Marquette University

e-Publications@Marquette

Biological Sciences Faculty Research and

Publications

Biological Sciences, Department of

$10-2016$

\title{
Destabilization of The Ornithine Decarboxylase mRNA Transcript by the RNA-Binding Protein Tristetraprolin
}

\author{
Shannon L. Nowotarski \\ Pennsylvania State University - Berks \\ Sofia Origanti \\ Marquette University, sofia.origanti@marquette.edu \\ Suzanne Sass-Kuhn \\ Pennsylvania State University - College of Medicine \\ Lisa M. Shantz \\ Pennsylvania State University - College of Medicine
}

Follow this and additional works at: https://epublications.marquette.edu/bio_fac

Part of the Biology Commons

\section{Recommended Citation}

Nowotarski, Shannon L.; Origanti, Sofia; Sass-Kuhn, Suzanne; and Shantz, Lisa M., "Destabilization of The Ornithine Decarboxylase mRNA Transcript by the RNA-Binding Protein Tristetraprolin" (2016). Biological Sciences Faculty Research and Publications. 536.

https://epublications.marquette.edu/bio_fac/536 
Marquette University

e-Publications@Marquette

\section{Department of Biology Faculty Research and Publications/College of Arts and Sciences}

This paper is NOT THE PUBLISHED VERSION.

Access the published version at the link in the citation below.

Amino Acids, Vol. 48, No. 10 (October 2016): 2303-2311. DOI. This article is (C Springer and permission has been granted for this version to appear in e-Publications@Marquette. Springer does not grant permission for this article to be further copied/distributed or hosted elsewhere without the express permission from Springer.

\section{Destabilization of the Ornithine Decarboxylase mRNA Transcript by the RNA- Binding Protein Tristetraprolin}

Shannon L. Nowotarski

Division of Science, The Pennsylvania State University Berks Campus, Reading, PA

Sofia Origanti

Department of Biological Sciences, Marquette University, Milwaukee, WI

Suzanne Sass-Kuhn

Department of Cellular and Molecular Physiology, The Pennsylvania State University College of Medicine, Hershey, PA

Lisa M. Shantz

Department of Cellular and Molecular Physiology, The Pennsylvania State University College of Medicine, Hershey, PA

\section{Abstract}

Ornithine decarboxylase (ODC) is the first and usually rate-limiting enzyme in the polyamine biosynthetic pathway. In a normal physiological state, ODC is tightly regulated. However, during neoplastic transformation, 
ODC expression becomes upregulated. The studies described here show that the ODC mRNA transcript is destabilized by the RNA-binding protein tristetraprolin (TTP). We show that TTP is able to bind to the ODC mRNA transcript in both non-transformed RIE-1 cells and transformed Ras $12 \mathrm{~V}$ cells. Moreover, using mouse embryonic fibroblast cell lines that are devoid of a functional TTP protein, we demonstrate that in the absence of TTP both ODC mRNA stability and ODC enzyme activity increase when compared to wild-type cells. Finally, we show that the ODC $3^{\prime}$ untranslated region contains cis acting destabilizing elements that are affected by, but not solely dependent on, TTP expression. Together, these data support the hypothesis that TTP plays a role in the posttranscriptional regulation of the ODC mRNA transcript.

\section{Introduction}

Ornithine decarboxylase (ODC) is the first biosynthetic enzyme in the polyamine pathway and converts the amino acid ornithine to the diamine putrescine, which is subsequently converted to the higher polyamines spermidine and spermine, respectively (Nowotarski et al. 2015; Pegg 2006). Under normal physiological conditions, ODC intracellular levels are tightly regulated through transcriptional and translational control and protein degradation (Bello-Fernandez et al. 1993; Shantz and Pegg 1999; Wallon et al. 1995; Zhao and Butler 2001). However, it has been well documented that an increased activity of ODC correlates with a variety of malignancies, including breast, colon, and skin cancers (Gerner and Meyskens 2004; Nowotarski et al. 2013, 2015). Particularly in the skin, the link between elevated ODC enzyme activity and tumor formation is indisputable, thereby making ODC an attractive chemoprevention target for combating non-melanoma skin cancer (Feith et al. 2005; Nowotarski et al. 2015; O’Brien 1976; Smith et al. 1997).

Changes in the intracellular polyamine pools have been shown to affect the localization of RNA-binding proteins (RBPs), which can influence the stability and translational efficiency of their target mRNA transcripts (Rao and Wang 2010). These mechanisms provide a rapid means of regulating gene expression. RBPs have been classically shown to bind to the $3^{\prime}$ untranslated region (UTR) of labile transcripts (Parker and Sheth 2007). Three categories of RBPs have been defined: those that stabilize mRNA, those that destabilize mRNA, and those that can both stabilize and destabilize mRNA (Guhaniyogi and Brewer 2001). Moreover, the physiological relevance of mRNA stability has been highlighted as an underlying factor in a variety of disease states (Benjamin and Moroni 2007; Lopez de Silanes et al. 2007). One such trans-acting RBP is tristetraprolin (TTP).

TTP is one of the three widely distributed RBPs that contain Cys-Cys-Cys-His-zinc (CCCH-zinc) finger RNAbinding domains. Other family members of TTP include butyrate response factor 1 (BRF1) and BRF2. These RBPs classically bind to UUAUUUUAUUU sequences in the 3'UTR of the target message (Brewer et al. 2004). TTP binds to, and destabilizes, numerous transcripts, including TNF- $\alpha$, GM-CSF, VEGF, and IL-3 (Carballo et al. 2000; Lai et al. 1999; Stoecklin et al. 2000; Suswam et al. 2008; Taylor et al. 1996a). Moreover, TTP interacts with proteins that are associated with RNA decay, such as DCP1, DCP2, XRN1, and CCR4, and has been shown to stimulate mRNA deadenylation and promote mRNA decay (Brooks and Blackshear 2013; Fenger-Gron et al. 2005). Similar to the stabilizing RBP HuR, TTP has been found to shuttle rapidly from the nucleus to the cytoplasm in response to serum and phorbol esters (Taylor et al. 1996b). In response to cellular stress, TTP has been shown to localize to stress granules and P-bodies, and it is hypothesized that this functions to relocate stalled mRNA to the mRNA decay machinery (Kedersha et al. 2005; Stoecklin et al. 2004).

Studies show that TTP expression is lower in tumor samples when compared to non-tumorigenic tissue samples (Brennan et al. 2009). In an array study using a variety of epithelial cancer cDNA samples, as well as nontransformed controls, TTP mRNA levels were shown to be decreased in $65 \%$ of tumors when compared to nontransformed controls. The reduction in TTP mRNA was frequent in thyroid, breast, lung, ovary, and uterine tumors. Western blot analysis confirmed that TTP protein expression was also suppressed in tumor samples 
(Brennan et al. 2009). These data suggest that TTP expression is frequently repressed in epithelial tumors and correlates TTP expression with the tumor development.

Previously, we demonstrated that ODC is regulated at the level of RNA stability in both keratinocyte-derived cells and rat intestinal epithelial (RIE-1) cells in part by binding to HuR, which stabilizes ODC mRNA in cells that have undergone neoplastic transformation (Nowotarski and Shantz 2010; Origanti et al. 2012). The work described here shows that TTP also binds to the ODC transcript in parental RIE-1 cells, and in cells that are transformed by $\mathrm{H}$-Ras12V expression. Using cells from TTP knockout mice, we demonstrate that the absence of TTP correlates with increased ODC enzyme activity as well as a stabilization of ODC mRNA. Further studies suggest that the ODC 3' UTR also contains both TTP-dependent and TTP-independent negative regulatory elements. Moreover, we show using an in vivo model of non-melanoma skin cancer progression that TTP expression is reduced as tumor grade progresses. Thus, these studies add to our understanding of ODC posttranscriptional regulation by RBPs, and highlight TTP as a trans-acting element that influences ODC posttranscriptional regulation.

\section{Materials and methods}

\section{Cell culture}

RIE-1 cells (a generous gift from Dr. Kenneth Brown, The Barbraham Institute, Cambridge, UK) were maintained in Dulbecco's modified Eagle's medium (DMEM) supplemented with $10 \%$ fetal bovine serum (FBS) and $1 \%$ penicillin/streptomycin. Ras12V cells were generated from RIE-1 cells as described previously (Origanti and Shantz 2007). For transfections, either pcDNA3-FLAG-TTP (a generous gift of Dr. Jens Lykke-Anderson, University of Colorado) or empty vector control (Life Technologies, Carlsbad, CA) was transiently transfected into Ras $12 \mathrm{~V}$ cells using Lipofectamine 2000 (Life Technologies), and cells were harvested $48 \mathrm{~h}$ later. $\mathrm{TTP}^{+/+}$and TTP ${ }^{-/-}$mouse embryonic fibroblasts (MEFs, a generous gift from Dr. Perry J. Blackshear, NIEHS, Durham, NC) derived from E14.5 mouse embryos were cultured in DMEM supplemented with $10 \% \mathrm{FBS}$ and $1 \%$ penicillin/streptomycin. These cells have been described previously (Lai et al. 2006). All cell culture stock flasks were incubated at $37^{\circ} \mathrm{C}$ in a humidified atmosphere of $95 \%$ air, $5 \% \mathrm{CO}_{2}$.

\section{Animal treatment}

All experiments involving mice were carried out in compliance with the Guide for the Care and Use of Laboratory Animals, and protocols approved by the Animal Care and Use Committee of the Pennsylvania State University College of Medicine. For mouse experiments, the dorsal surface of FVB/N mice (6-8 weeks) was treated with a single application of DMBA followed by multiple applications of TPA (25 weeks, 2X/week) to induce tumors, as described previously (Carr et al. 2012). Animals were euthanized, and dorsal skin was treated with a depilatory agent for $3 \mathrm{~min}$, washed, and tumors were removed. Tumor samples were placed into ice-cold 1X RIPA buffer (20 mM Tris- $\mathrm{HCl}$ pH 7.5, $150 \mathrm{mM} \mathrm{NaCl}, 1$ mM Na 2 EDTA, 1 mM EGTA, 1 \% NP-40, 1 \% sodium deoxycholate, $2.5 \mathrm{mM}$ sodium pyrophosphate, $1 \mathrm{mM}$-beta-glycerophosphate, $1 \mathrm{mM} \mathrm{Na}_{3} \mathrm{VO}_{4}, 1 \mu \mathrm{g} / \mathrm{ml}$ leupeptin, $1 \mathrm{mM} \mathrm{PMSF}$, and $1 \mathrm{X}$ protease inhibitor cocktail) (Santa Cruz Biotechnology, Santa Cruz, CA). Following sonication, samples were centrifuged, and the supernatants were collected. Samples were flash-frozen and later processed in RIPA buffer by homogenizing for $30 \mathrm{~s}$ on ice using a Polytron homogenizer, tissue was centrifuged at $30,000 \times g$ for $30 \mathrm{~min}$ at $4{ }^{\circ} \mathrm{C}$, and supernatants were collected. Equal amounts of protein were then subjected to SDS-PAGE.

\section{Western blot analysis}

Western blotting was conducted as described previously (Origanti and Shantz 2007). Membranes were probed using antibodies that recognized TTP (EMD Millipore/Merck KGaA, Germany), HuR, or GAPDH (Santa Cruz Biotechnology). These antibodies were used at a 1:1000 dilution. A polyclonal antibody to ODC was prepared as 
described previously (Shantz 2004). Western blots were quantitated using the Syngene software (Syngene, Frederick, MD).

\section{Assay for ODC enzyme activity}

ODC enzyme activity was determined by a radiometric assay, measuring the amount of ${ }^{14} \mathrm{CO}_{2}$ released from each reaction in which $\mathrm{L}-\left[1-{ }^{14} \mathrm{C}\right]$ ornithine is enzymatically converted to ${ }^{14} \mathrm{CO}_{2}$ by ornithine decarboxylase. Collection and analysis were carried out, as described previously (Coleman and Pegg 1998).

\section{RNA stability using Actinomycin D assay and qRT-PCR}

Cells were grown to $70 \%$ confluency and treated with $10-\mu \mathrm{g} / \mathrm{ml}$ Actinomycin D (Sigma-Aldrich, St. Louis, MO). RNA was harvested using the TRIzol reagent according to the manufacturer's instructions (Invitrogen) at 0, 4, and $8 \mathrm{~h}$ post-Actinomycin $\mathrm{D}$ treatment. Total RNA was then used in reverse transcription and PCR amplification. qRT-PCR was performed using the Step One Plus Real-Time PCR system (Applied Biosystems), and the following primers: ODC sense, 5'-CGAGAACCATGAGCAGCTTTAC-3'; ODC antisense, 5'-GCATCCTTATCGTCAGAGGAAG-3' (annealing at $58^{\circ} \mathrm{C}$ ); Cyclophilin A sense, 5'-GCAGGTCCATCTACGGAGAG-3'; and Cyclophilin A antisense, 5'CTGGGAACCGTTGTGTTTGG-3' (annealing at $58^{\circ} \mathrm{C}$ ). To quantify relative gene expression, the comparative cycle threshold $\left(C_{\mathrm{t}}\right)$ method was utilized, and the $C_{\mathrm{t}}$ values for the gene of interest were normalized to the $C_{\mathrm{t}}$ values of Cyclophilin A, and were presented in relation to the untreated ( $0 \mathrm{~h}$ time point) controls. Analysis was performed on the Step One Plus Real-Time PCR software (Applied Biosystems).

\section{Biotin-labeled RNA protein-binding assays}

A synthetic ODC transcript was generated by isolating total RNA from Ras $12 \mathrm{~V}$ cells and then using reverse transcriptase to produce cDNA. The cDNA was used as a template for PCR amplification of the full-length ODC $3^{\prime}$ UTR. The sense primers for each reaction contained the T7 RNA polymerase promoter sequence on the $5^{\prime}$ end $\left(T_{7}\right)$ : 5'-CCAAGCTTCTAATACGACTCACTATAGGGAGA-3' to allow further amplification. The PCR templates were used to transcribe biotinylated RNAs using the T7 polymerase and biotin-labeled CTP (Invitrogen) as reported previously (Zou et al. 2006). The resulting biotin-labeled transcripts $(15 \mu \mathrm{l})$ were incubated with $120 \mu \mathrm{g}$ of cytoplasmic lysate for $30 \mathrm{~min}$ at room temperature. The RNA-protein complexes were then harvested using paramagnetic streptavidin-conjugated Dynabeads (Invitrogen), and were analyzed by Western blot analysis using the TTP antibody described above or an HuR antibody (Santa Cruz Biotechnology).

\section{RNP (ribonucleoprotein) immunoprecipitation}

For immunoprecipitation (IP) of endogenous RNA-protein-binding complexes, $2000 \mu \mathrm{g}$ of cytoplasmic lysate was incubated for $1 \mathrm{~h}$ at room temperature with a 100- $\mu$ l suspension of protein-A SureBeads (BioRad, Hercules, CA) that had been precoated with $15 \mu \mathrm{g}$ of either rabbit IgG (Cell Signaling, Danvers, MA) or TTP antibody (Millipore) as described (Nowotarski et al. 2011). The RNA IP material was analyzed by washing the beads with PBS-Tween buffer $(0.05 \%$ Tween-20) and further incubated with $0.05-\mathrm{mg} / \mathrm{ml}$ proteinase $\mathrm{K}, 10 \%$ SDS, and $100-\mu \mathrm{l}$ PBS-Tween for $30 \mathrm{~min}$ at $55^{\circ} \mathrm{C}$. RNA was precipitated using phenol-chloroform extraction in the presence of glycoblue (Invitrogen). RNA from the IP was then reverse transcribed using Superscript VILO (Invitrogen) to detect the presence of ODC mRNA. qRT-PCR was performed using the Step One Plus Real-Time PCR system (Applied Biosystems), and the following primers: ODC sense, 5'-CGAGAACCATGAGCAGCTTTAC-3'; ODC antisense, 5'CTACACATTGATCCTAGCAGAA-3' (annealing at $58^{\circ} \mathrm{C}$ ); GAPDH sense, $5^{\prime}$-CCAGCCTCGTCCCGTAGACAAAA-3'; and GAPDH antisense, $5^{\prime}$-TCGGCCTTGACTGTGCCGTTGAA-3'. To quantify relative gene expression, the comparative cycle threshold $\left(C_{t}\right)$ method was utilized, and the $C_{t}$ values for the gene of interest were normalized to the $C_{\mathrm{t}}$ values of GAPDH, and were presented in relation to the rabbit IgG controls. Analysis was performed on the Step One Plus Real-Time PCR software (Applied Biosystems). 


\section{Luciferase assays}

The ODC 3'UTR was cloned into the pGL3-Control vector (Promega, Madison, WI), and placed downstream of the Firefly luciferase reporter gene. This plasmid is denoted as pODC3'UTRLuc. To investigate the influence of the ODC $3^{\prime} U T R$ on luciferase activity, $T \mathrm{P}^{+/+}$and $\mathrm{TP}^{-/-}$cells were transfected with either $2 \mu \mathrm{g}$ of pODC3'UTRLuc or the pGL3 Control using Lipofectamine 2000 transfection reagent as per the manufacturer's instructions (Life Technologies). Mock-transfected cells were treated with Lipofectamine 2000 only. The pRL-SV40 Renilla reporter plasmid was co-transfected at $0.2 \mu \mathrm{g}$ per plate to act as a transfection efficiency control (Promega). Fourty-eight $\mathrm{h}$ after post-transfection, cells were harvested in $1 \mathrm{X}$ Passive Lysis Buffer (Promega) and assayed using the DualLuciferase Kit as per the manufacturer's instructions (Promega). For each sample, the Firefly luciferase activity was normalized to the Renilla luciferase activity, and the data are expressed as the Firefly/Renilla ratio. The data were normalized to the pGL3 Control.

\section{Statistics}

The results are expressed as the means \pm standard errors (SE). Statistical analysis was performed using Student's $t$-test. $P$ values of $<0.05$ were considered significant.

\section{Results}

TTP is elevated in non-transformed cells and is able to bind to a synthetic ODC 3'UTR in both non-transformed and transformed cells

Previous work in our lab has shown that ODC is post-transcriptionally regulated by the RBP HuR. We demonstrated that the ODC 3'UTR contains numerous AU-rich elements, which are thought to be target sites for not only HuR but also other RBPs (Nowotarski and Shantz 2010). Furthermore, we showed that HuR is capable of binding to deletion mutants of the ODC 3'UTR that are significantly less stable than the wild-type 3'UTR (Origanti et al. 2012). These results suggest that other RBPs may be competing with HuR for binding to a given target within the ODC transcript. One of the most common HuR-binding competitors is TTP (Blaxall et al. 2000; Gardiner et al. 2015; Ming et al. 2001). Therefore, a biotinylated pulldown assay was conducted in both nontransformed RIE-1 cells and Ras12V transformed cells to determine whether TTP could bind to synthetic transcripts corresponding to the full-length ODC 3'UTR. The data show that TTP binds to the ODC $3^{\prime} U T R$ in both RIE-1 cells and Ras12V cells (Fig. 1a). Interestingly, TTP actually associates slightly more strongly with the ODC transcript in the transformed Ras12V cells (Fig. 1a). However, when the same cell extract was analyzed for HuR association with the ODC 3'UTR, there was a massive increase in HuR association to the ODC 3'UTR in Ras12V cells (Fig. 1a). In fact, the ratio of HuR bound to TTP bound increased more than ten-fold in Ras12V cells compared to RIE-1 cells. Analysis of whole cell lysates revealed that TTP protein levels are higher in RIE-1 cells than in Ras12V cells (Fig. 1b), and our previous results showed that HuR protein levels were higher in Ras12V cells when compared to RIE-1 cells (Origanti et al. 2012). To determine the effect of increasing TTP in Ras12V cells, we overexpressed a FLAG-TTP plasmid. The results show that cells overexpressing TTP contained $40 \%$ less ODC protein compared to cells transfected with an empty vector control (Fig. 1c), consistent with destabilization of the ODC transcript by TTP. 


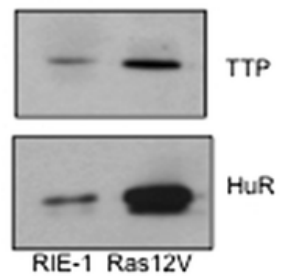

b

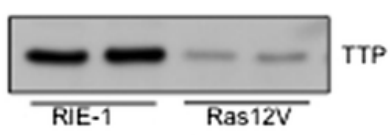

c
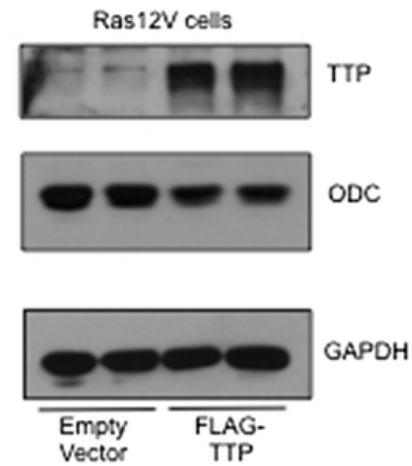

Fig. 1. TTP is able to bind to synthetic ODC $3^{\prime} U T R$ transcripts in both non-transformed RIE-1 cells and transformed Ras $12 \mathrm{~V}$ cells. a Cytoplasmic lysates $(120 \mu \mathrm{g})$ prepared from RIE-1 or Ras $12 \mathrm{~V}$ cell lines were incubated with $15 \mu \mathrm{l}$ of synthetic biotin-labeled ODC $3^{\prime} U T R$ transcript for $30 \mathrm{~min}$ at room temperature. The resulting ribonucleoprotein complexes were pulled down using streptavidin beads, and the presence of TTP and HuR was assessed via Western blot analysis. This experiment was done in duplicate with similar results. b Whole cell lysates were prepared in 1X RIPA buffer. TTP was detected via Western blot analysis. This experiment was conducted in duplicate with similar results. c Flag-TTP plasmid or empty vector was transiently transfected into Ras12V cells, and TTP, ODC, and GAPDH were analyzed by Western blot analysis. This experiment was conducted in duplicate with similar results

\section{TTP binds to endogenous ODC mRNA in wild-type MEFs}

To more fully understand the results in RIE-1 cells, we used MEFs derived from TTP knockout mice. The TTP protein in these cells is not truly knocked out, but rather is truncated and, therefore, non-functional (Taylor et al. 1996a). The intracellular association of ODC mRNA and TTP was assessed via an RNA immunoprecipitation (RNA IP) assay that allows measurement of the endogenous association of TTP and its target mRNAs. Previous studies have shown that TTP association with target RNA can range from 12.5-fold to 60 -fold enrichment in wildtype MEFs; although in these studies, cells were treated with budesonide to induce TTP (Ishmael et al. 2008). Our results show an association of TTP with ODC mRNA in wild-type MEF cytoplasmic lysates, and quantitation using qRT-PCR analysis revealed a 3.9-fold increase in ODC mRNA in TTP pulldown material when compared to the IgG control (Fig. 2). 


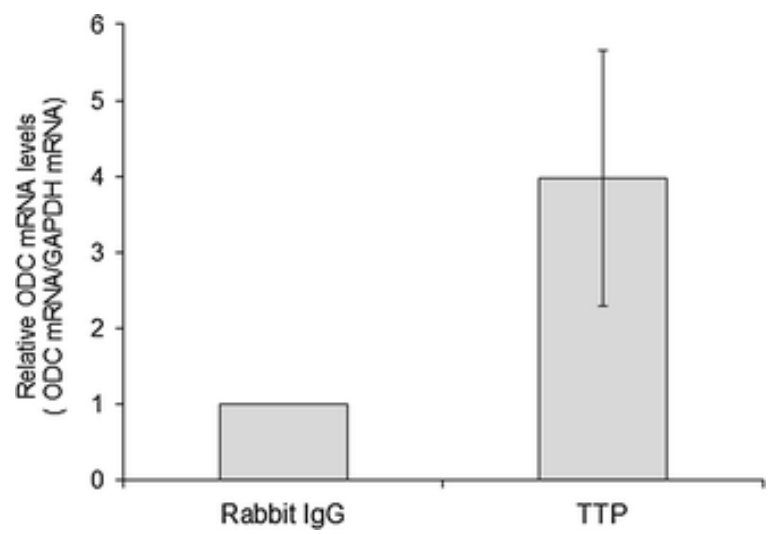

Fig. 2 TTP associates with endogenous ODC mRNA in wild-type MEF cells. Cytoplasmic lysates from wild-type MEFs were used for IP with anti-TTP or nonspecific rabbit IgG. RNA in the IP material was extracted using phenol chloroform extraction, used to make CDNA, and analyzed for the presence of ODC transcript using primers that were specific for the ODC coding region. The data were analyzed via qRT-PCR. The values are the mean $\pm S E$ from three separate experiments. $P=0.127$

ODC mRNA stability and activity are increased in TTP knockout MEFs when compared to wild-type MEFs

We next wanted to determine the effect of knocking out TTP on ODC mRNA stability. In agreement with the RNA IP experiment discussed above, ODC stability increased four-fold in TTP knockout MEFs when compared to wildtype MEFs (Fig. 3a). Interestingly, TTP wild-type MEFs displayed a two-fold higher level of steady-state ODC mRNA compared to TTP knockout MEFs (data not shown). These data suggest that ODC is post-transcriptionally regulated in these cells at the level of translational efficiency or transcript degradation. To determine whether a reduction in ODC mRNA stability correlated with a decrease in ODC enzyme activity, we assessed the ODC enzyme activity in both wild-type and TTP knockout MEFs. A statistically significant reduction (two-fold) in ODC enzyme activity was observed in log-phase wild-type cells when compared to TTP knockout cells (Fig. 3b).

a
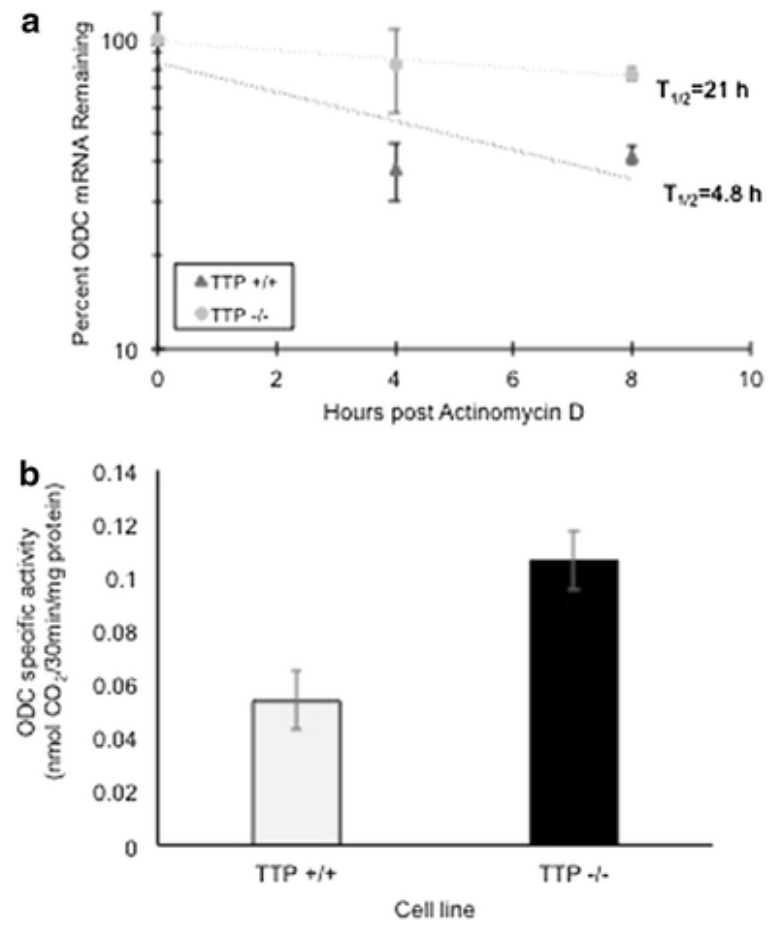
Fig. 3 ODC mRNA stability and ODC enzyme activity are increased in TTP knockout MEFs when compared to wildtype MEFs. a TTP wild-type (+/+) and TTP knockout cells (-/-) were used. ODC mRNA half-life was determined using $10 \mu \mathrm{g}$ of Actinomycin $\mathrm{D} / \mathrm{ml}$. Total RNA was isolated at the indicated times, and the levels of ODC mRNA and GAPDH mRNA were assessed via qRT-PCR, as described in the "Materials and methods". The results are shown as the mean \pm SE from three separate experiments. $\mathbf{b}$ TTP wild-type (+/+) and TTP knockout (-/-) cells were harvested in ODC harvest buffer. An ODC enzyme activity assay was conducted as described in the "Materials and methods" section. This experiment was conducted five times $(n=17)$. A student's $t$-test was conducted on the data. ${ }^{*} P<0.05$ was considered statistically significant when comparing TTP $(+/+)$ cells ODC enzyme activity to TTP (-/-) cells ODC enzyme activity. $P=0.0170$

To investigate the regulation of ODC by its $3^{\prime} U T R$, we used transient transfections of luciferase reporters. Expression of a luciferase reporter vector containing the ODC3'UTR was lower in TTP wild-type cells compared to TTP knockouts, suggesting that TTP does play a role in mediating the luciferase expression (Fig. 4). When these cells were each compared to their respective empty vector controls, insertion of the ODC $3^{\prime} U T R$ into the pGL3 vector (pODC3'UTRLuc) resulted in a $75 \%$ reduction in luciferase activity in TTP wild-type cells and a $50 \%$ reduction in luciferase activity in TTP knockout cells, suggesting the presence of a negative regulatory element in the $3^{\prime} U T R$ that is not solely dependent on TTP expression (Fig. 4).

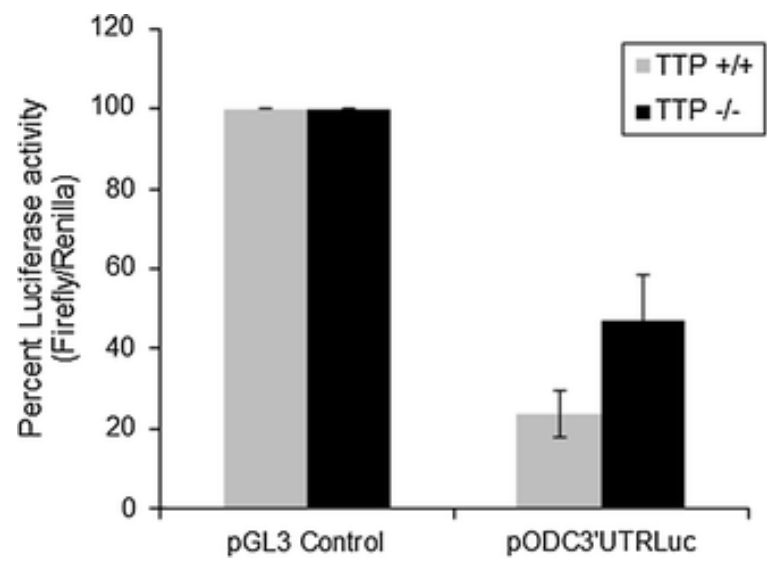

Fig. 4 ODC $3^{\prime}$ UTR causes a decrease in luciferase reporter activity in MEF cells. TTP wild-type (+/+) and TTP knockout (-/-) cells were transfected with either pGL3 control or pODC3'UTRLuc as described in the "Materials and methods" section. Fourty-eight h after transfection, cells were assayed for luciferase activity. Firefly luciferase activity was normalized to Renilla activity for each sample. The luciferase activity for the pGL3 control was set to $100 \%$. Cells transfected with pODC3'UTRLuc are shown as a percent of pGL3 control activity. Values are mean $\pm \mathrm{SE}(n=7)$

\section{TTP protein levels decrease during non-melanoma skin cancer progression}

We used an in vivo mouse model of NMSC to determine the levels of TTP during tumor progression. When we examined skin tumors harvested from mice treated with DMBA/TPA for 25 weeks, we saw that as tumor grade increases from benign papilloma to squamous cell carcinoma, the level of TTP protein decreases modestly

(Fig. 5). We did not see consistent levels of our GAPDH loading control across samples in these experiments; perhaps, due to the heterogeneity of the tumor tissue being analyzed. The utilization of other loading controls did not alter the relative ratios of TTP protein normalized to a loading control (data not shown), suggesting that the changes in TTP observed are real. The data shown agree with results published previously suggesting that TTP decreases during tumor progression (Brennan et al. 2009). Since ODC protein is known to be induced in skin tumors (Gilmour 2007; Gilmour et al. 1985), an increase in ODC mRNA stability brought about by changes in TTP binding may play a role in this process. 

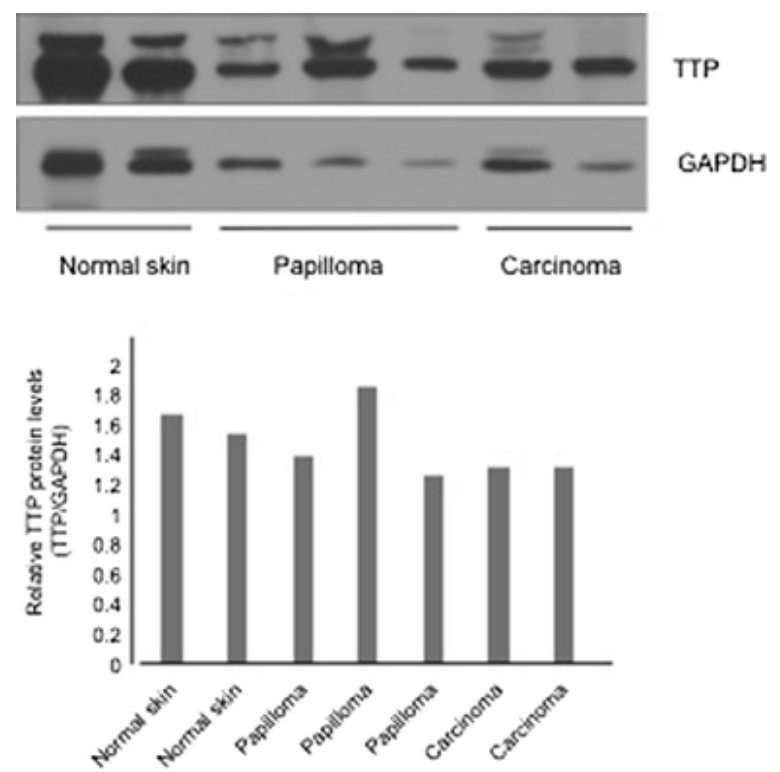

Fig. 5 TTP expression is reduced as tumor grade increases during NMSC progression. FVB/N mice were treated with DMBA and TPA (25 weeks, 2X/week), as described in the "Materials and methods" section. Tissue samples were run for the presence of TTP using Western blot analysis. Blots were stripped and probed for GAPDH. Western blots were quantified using the SynGene software. Papilloma denotes benign papillomas and carcinoma denotes squamous cell carcinomas. The representative blot shows two or three samples from different mice

\section{Discussion}

Our studies show that cytoplasmic TTP binds to a synthetic ODC 3'UTR in both non-transformed RIE-1 cells as well as transformed Ras12V cells (Fig. 1a). Moreover, we show that TTP is able to bind to endogenous ODC message in these cells (Fig. 2). A functional correlation between TTP and ODC mRNA stability and ODC enzyme activity was shown using TTP knockout MEFs (Fig. 3a, b), and the ablation of a functional TTP protein results in both a dramatic elevation in ODC mRNA stability, (Fig. 3a) as well as a significant increase in ODC enzyme activity (Fig. 3b). Experiments analyzing the influence of the ODC $3^{\prime} U T R$ on luciferase activity in our TTP wild-type and TTP knockout cell lines revealed that the ODC 3'UTR contains a negative regulatory element that is influenced, but not solely dependent on TTP expression, as the insertion of the $3^{\prime} U T R$ into the PGL3 control vector showed a dramatic decrease in the level of luciferase activity in both TTP wild-type and TTP knockout MEFs (Fig. 4). These data are in agreement with previous results shown by Lee et al., in which the $3^{\prime} U T R$ of VEGF was fused to the luciferase gene in the pGL3 plasmid. When TTP was overexpressed in Colo320 cells, the luciferase activity was markedly reduced in comparison to the control pGL3 plasmid, suggesting that TTP is a negative regulator of UTRmediated luciferase activity (Lee et al. 2009). Taken together, these data suggest that the RBP TTP is not only able to bind to the ODC $3^{\prime} U T R$, but also that the binding of TTP to the ODC $3^{\prime} U T R$ destabilizes the ODC mRNA message and reduces the overall expression of the ODC protein as measured by ODC enzyme activity assays.

TTP, a CCH-zinc finger RBP, has been shown to bind to and decrease the mRNA stability of numerous transcripts (Brooks and Blackshear 2013). Furthermore, in a study looking at TTP expression in colon cancer, it was found that TTP levels were dramatically decreased in adenocarcinoma samples when compared to non-tumorigenic controls. The level of TTP had an inverse relationship with a marker of neoplastic transformation, VEGF. VEGF protein levels were found to be elevated in adenocarcinoma samples and low in controls. TTP overexpression in Colo320 cells, a human colorectal cell line, correlated with a marked decrease in mRNA stability of VEGF, a decrease in cell proliferation, and smaller tumors when these cells were injected into nude mice. These studies demonstrate that TTP negatively regulates tumorigenesis, and highlight TTP as a possible target for 
chemoprevention and chemotherapy (Lee et al. 2009). Our results support previous findings that TTP decreases during tumor progression; (Brennan et al. 2009) by showing that in an NMSC mouse model, we see a trend towards reduction in TTP expression as tumor grade increases; however, these data were not statistically significant (Fig. 5). Interestingly, we observed the presence of a TTP doublet in many of our samples. This doublet may represent a post-translationally modified species of TTP, as TTP can be extensively phosphorylated yielding a size range of 37-50 kD. Either reduction or hyperphosphorylation of the TTP protein has been correlated with a transformed phenotype in numerous cancers, including breast, ovarian, and malignant glioma (Brennan et al. 2009; Suswam et al. 2008). Thus, more studies investigating the over 20 putative phosphorylation sites on TTP need to be conducted to determine both the expression and the physiological relevance of these modifications in our system.

Recently, it has been shown that TTP impairs Myc-induced lymphoma. These studies revealed that Myc regulates hundreds of ARE-containing genes and RBPs, including TTP, and TTP suppression was shown to be a hallmark of cancers, in which Myc was directly involved in tumorigenesis. The restoration of TTP expression compromised Myc-induced tumorigenesis, suggesting that TTP not only acts as a tumor suppressor but also that Myc targets various RBPs and ARE-containing transcripts to control neoplastic transformation (Rounbehler et al. 2012). These studies are interesting; because, in addition to regulating the RBP TTP, it has been well documented that Myc is able to regulate the transcription of Odc (Auvinen et al. 2003; Bello-Fernandez et al. 1993; Erdman et al. 1999). However, in the case of Odc, Myc acts as a positive regulator of gene expression. These previous studies in addition to our current data suggest a complex network of regulation for ODC and further support the notion that ODC is rational target for chemoprevention and/or chemotherapeutics.

The current studies demonstrate for the first time that ODC is not only post-transcriptionally regulated by the stabilizing RBP HuR (Nowotarski and Shantz 2010), but is also a target for the destabilizing RBP TTP. These studies reveal a dynamic and complex process of regulation of Odc in both normal cellular physiology and pathophysiology. Since ODC mRNA is bound by the stabilizing RBP HuR in a malignant state, one may not expect a destabilizing protein, such as TTP to bind to the ODC 3'UTR in Ras12V cells. However, we observed that both HuR and TTP bind to the ODC message in Ras $12 \mathrm{~V}$ cells, suggesting that both stabilizing and destabilizing RBPs play a role in message regulation in transformed cells. In agreement with this, Lal et al. (2004) showed that HuR and the destabilizing RBP AUF1 bound to the $3^{\prime}$ UTRs of p21 and Cyclin D1 at different non-overlapping regions, suggesting that RBPs can bind to the same mRNA simultaneously. Experiments investigating the competition of destabilizing and stabilizing RBPs on the ODC transcript are ongoing, as are experiments determining the cis and trans-acting elements on the ODC 3'UTR which influence luciferase expression.

In conclusion, we describe a novel trans-acting mechanism of ODC regulation. We demonstrate, using a genetic approach, that ODC mRNA stability and ODC enzyme activity are increased in cells, in which a functional TTP protein has been ablated. These data suggest that the RBP TTP not only binds to the ODC mRNA transcript, but also that this interaction between the TTP protein and ODC mRNA transcript is functionally relevant. These studies thus add to our knowledge of ODC regulation at the level of mRNA stability.

\section{References}

Auvinen $\mathrm{M}$ et al (2003) Transcriptional regulation of the ornithine decarboxylase gene by c-Myc/Max/Mad network and retinoblastoma protein interacting with c-Myc. Int J Biochem Cell Biol 35:496-521

Bello-Fernandez C, Packham G, Cleveland JL (1993) The ornithine decarboxylase gene is a transcriptional target of c-Myc. Proc Natl Acad Sci USA 90:7804-7808

Benjamin D, Moroni C (2007) mRNA stability and cancer: an emerging link? Expert Opin Biol Ther 7:1515-1529. doi:10.1517/14712598.7.10.1515 
Blaxall BC, Dwyer-Nield LD, Bauer AK, Bohlmeyer TJ, Malkinson AM, Port JD (2000) Differential expression and localization of the mRNA binding proteins, AU-rich element mRNA binding protein (AUF1) and Hu antigen $\mathrm{R}$ (HuR), in neoplastic lung tissue. Mol Carcinog 28:76-83

Brennan SE, Kuwano Y, Alkharouf N, Blackshear PJ, Gorospe M, Wilson GM (2009) The mRNA-destabilizing protein tristetraprolin is suppressed in many cancers, altering tumorigenic phenotypes and patient prognosis. Cancer Res 69:5168-5176. doi:10.1158/0008-5472.CAN-08-4238

Brewer BY, Malicka J, Blackshear PJ, Wilson GM (2004) RNA sequence elements required for high affinity binding by the zinc finger domain of tristetraprolin: conformational changes coupled to the bipartite nature of Au-rich MRNA-destabilizing motifs. J Biol Chem 279:27870-27877. doi:10.1074/jbc.M402551200

Brooks SA, Blackshear PJ (2013) Tristetraprolin (TTP): interactions with mRNA and proteins, and current thoughts on mechanisms of action. Biochim Biophys Acta 1829:666-679. doi:10.1016/j.bbagrm.2013.02.003

Carballo E, Lai WS, Blackshear PJ (2000) Evidence that tristetraprolin is a physiological regulator of granulocytemacrophage colony-stimulating factor messenger RNA deadenylation and stability. Blood 95:1891-1899

Carr TD, DiGiovanni J, Lynch CJ, Shantz LM (2012) Inhibition of mTOR suppresses UVB-induced keratinocyte proliferation and survival. Cancer Prev Res (Phila) 5:1394-1404. doi:10.1158/1940-6207.CAPR-12-0272-T

Coleman CS, Pegg AE (eds) (1998) Assay of mammalian ornithine decarboxylase activity using $\left[{ }^{14} \mathrm{C}\right]$-ornithine vol 79. Polyamine protocols. Humana Press Inc., Totowa

Erdman SH, Ignatenko NA, Powell MB, Blohm-Mangone KA, Holubec H, Guillen-Rodriguez JM, Gerner EW (1999) APC-dependent changes in expression of genes influencing polyamine metabolism, and consequences for gastrointestinal carcinogenesis, in the Min mouse. Carcinogenesis 20:1709-1713

Feith DJ, Bol DK, Carboni JM, Lynch MJ, Sass-Kuhn S, Shoop PL, Shantz LM (2005) Induction of ornithine decarboxylase activity is a necessary step for mitogen-activated protein kinase kinase-induced skin tumorigenesis. Cancer Res 65:572-578

Fenger-Gron M, Fillman C, Norrild B, Lykke-Andersen J (2005) Multiple processing body factors and the ARE binding protein TTP activate mRNA decapping. Mol Cell 20:905-915. doi:10.1016/j.molcel.2005.10.031

Gardiner AS, Twiss JL, Perrone-Bizzozero NI (2015) Competing interactions of RNA-binding proteins, microRNAs, and their targets control neuronal development and function. Biomolecules 5:2903-2918. doi:10.3390/biom5042903

Gerner EW, Meyskens FL Jr (2004) Polyamines and cancer: old molecules, new understanding. Nat Rev Cancer 4:781-792. doi:10.1038/nrc1454

Gilmour SK (2007) Polyamines and nonmelanoma skin cancer. Toxicol Appl Pharmacol 224:249-256. doi:10.1016/j.taap.2006.11.023

Gilmour SK, Avdalovic N, Madara T, O'Brien TG (1985) Induction of ornithine decarboxylase by 12-Otetradecanoylphorbol 13-acetate in hamster fibroblasts. Relationship between levels of enzyme activity, immunoreactive protein, and RNA during the induction process. J Biol Chem 260:16439-16444

Guhaniyogi J, Brewer G (2001) Regulation of mRNA stability in mammalian cells. Gene 265:11-23

Ishmael FT et al (2008) Role of the RNA-binding protein tristetraprolin in glucocorticoid-mediated gene regulation. J Immunol 180:8342-8353

Kedersha $\mathrm{N}$ et al (2005) Stress granules and processing bodies are dynamically linked sites of mRNP remodeling. J Cell Biol 169:871-884. doi:10.1083/jcb.200502088

Lai WS, Carballo E, Strum JR, Kennington EA, Phillips RS, Blackshear PJ (1999) Evidence that tristetraprolin binds to AU-rich elements and promotes the deadenylation and destabilization of tumor necrosis factor alpha mRNA. Mol Cell Biol 19:4311-4323

Lai WS, Parker JS, Grissom SF, Stumpo DJ, Blackshear PJ (2006) Novel mRNA targets for tristetraprolin (TTP) identified by global analysis of stabilized transcripts in TTP-deficient fibroblasts. Mol Cell Biol 26:91969208. doi:10.1128/MCB.00945-06

Lal A, Mazan-Mamczarz K, Kawai T, Yang X, Martindale JL, Gorospe M (2004) Concurrent versus individual binding of HuR and AUF1 to common labile target mRNAs. EMBO J 23:3092-3102. doi:10.1038/sj.emboj.7600305 
Lee HH, Son YJ et al (2009) Tristetraprolin regulates expression of VEGF and tumorigenesis in human colon cancer. Int J Cancer 126:1817-1827

Lopez de Silanes I, Quesada MP, Esteller M (2007) Aberrant regulation of messenger RNA 3'-untranslated region in human cancer. Cell Oncol 29:1-17

Ming XF, Stoecklin G, Lu M, Looser R, Moroni C (2001) Parallel and independent regulation of interleukin-3 mRNA turnover by phosphatidylinositol 3-kinase and p38 mitogen-activated protein kinase. Mol Cell Biol 21:5778-5789

Nowotarski SL, Shantz LM (2010) Cytoplasmic accumulation of the RNA-binding protein HuR stabilizes the ornithine decarboxylase transcript in a murine nonmelanoma skin cancer model. J Biol Chem 285:31885-31894. doi:10.1074/jbc.M110.148767

Nowotarski SL, Origanti S, Shantz LM (2011) Polyamines: methods and protocols vol 270. Methods in molecular biology. Humana Press, Totowa

Nowotarski SL, Woster PM, Casero RA Jr (2013) Polyamines and cancer: implications for chemotherapy and chemoprevention. Expert Rev Mol Med 15:e3. doi:10.1017/erm.2013.3

Nowotarski SL, Feith DJ, Shantz LM (2015) Skin carcinogenesis studies using mouse models with altered polyamines. Cancer Growth Metastasis 8:17-27. doi:10.4137/CGM.S21219

O'Brien TG (1976) The induction of ornithine decarboxylase as an early, possibly obligatory, event in mouse skin carcinogenesis. Cancer Res 36:2644-2653

Origanti S, Shantz LM (2007) Ras transformation of RIE-1 cells activates cap-independent translation of ornithine decarboxylase: regulation by the Raf/MEK/ERK and phosphatidylinositol 3-kinase pathways. Cancer Res 67:4834-4842. doi:10.1158/0008-5472.CAN-06-4627

Origanti S, Nowotarski SL, Carr TD, Sass-Kuhn S, Xiao L, Wang JY, Shantz LM (2012) Ornithine decarboxylase mRNA is stabilized in an mTORC1-dependent manner in Ras-transformed cells. Biochem J 442:199-207. doi:10.1042/BJ20111464

Parker R, Sheth U (2007) P bodies and the control of mRNA translation and degradation. Mol Cell 25:635-646. doi:10.1016/j.molcel.2007.02.011

Pegg AE (2006) Regulation of ornithine decarboxylase. J Biol Chem 281:14529-14532

Rao JN, Wang JY (2010) In: Regulation of gastrointestinal mucosal growth. Morgan and Claypool Life Sciences, San Rafael, CA

Rounbehler RJ et al (2012) Tristetraprolin impairs myc-induced lymphoma and abolishes the malignant state. Cell 150:563-574. doi:10.1016/j.cell.2012.06.033

Shantz LM (2004) Transcriptional and translational control of ornithine decarboxylase during Ras transformation. Biochem J 377:257-264

Shantz LM, Pegg AE (1999) Translational regulation of ornithine decarboxylase and other enzymes of the polyamine pathway. Int J Biochem Cell Biol 31:107-122

Smith MK, Goral MA, Wright JH, Matrisian LM, Morris RJ, Klein-Szanto AJ, Gilmour SK (1997) Ornithine decarboxylase overexpression leads to increased epithelial tumor invasiveness. Cancer Res 57:21042108

Stoecklin G, Ming XF, Looser R, Moroni C (2000) Somatic mRNA turnover mutants implicate tristetraprolin in the interleukin-3 mRNA degradation pathway. Mol Cell Biol 20:3753-3763

Stoecklin G, Stubbs T, Kedersha N, Wax S, Rigby WF, Blackwell TK, Anderson P (2004) MK2-induced tristetraprolin:14-3-3 complexes prevent stress granule association and ARE-mRNA decay. EMBO J 23:1313-1324. doi:10.1038/sj.emboj.7600163

Suswam E et al (2008) Tristetraprolin down-regulates interleukin-8 and vascular endothelial growth factor in malignant glioma cells. Cancer Res 68:674-682. doi:10.1158/0008-5472.CAN-07-2751

Taylor GA et al (1996a) A pathogenetic role for TNF alpha in the syndrome of cachexia, arthritis, and autoimmunity resulting from tristetraprolin (TTP) deficiency. Immunity 4:445-454

Taylor GA, Thompson MJ, Lai WS, Blackshear PJ (1996b) Mitogens stimulate the rapid nuclear to cytosolic translocation of tristetraprolin, a potential zinc-finger transcription factor. Mol Endocrinol 10:140-146. doi:10.1210/mend.10.2.8825554 
Wallon UM, Persson L, Heby O (1995) Regulation of ornithine decarboxylase during cell growth. Changes in the stability and translatability of the mRNA, and in the turnover of the protein. Mol Cell Biochem 146:3944

Zhao B, Butler AP (2001) Core promoter involvement in the induction of rat ornithine decarboxylase by phorbol esters. Mol Carcinog 32:92-99

Zou T et al (2006) Polyamine depletion increases cytoplasmic levels of RNA-binding protein HuR leading to stabilization of nucleophosmin and p53 mRNAs. J Biol Chem 281:19387-19394. doi:10.1074/jbc.M602344200

\section{Acknowledgments}

Work in the authors' laboratory was funded by Grants from the National Institutes of Health (CA142051 and ES19242 to LMS).

\section{Ethics declarations}

\section{Research involving human participants and/or animals}

All applicable international, national, and/or institutional guidelines for the care and use of animals were followed. All experiments involving mice were carried out in compliance with the Guide for the Care and Use of Laboratory Animals, and protocols approved by the Animal Care and Use Committee of the Pennsylvania State University College of Medicine (Hershey, PA), the institution at which the studies were conducted.

\section{Human participation}

This article does not contain any studies with human participants performed by any of the authors.

\section{Conflict of interest}

The authors declare that they have no conflict of interest. 